\title{
VERIFICATION OF A SLIDING WINDOW PROTOCOL USING IOA AND MONA
}

\author{
Mark A. Smith* \\ IRISA \\ 35042 Rennes Cedex, France \\ Mark.Smith@irisa.fr
}

Nils Klarlund

ATET Labs Research

Florham Park, NJ 07932, U.S.A.

klarlund@research.att.com

\begin{abstract}
We show how to use a decision procedure for WS1S (the MONA tool) to give automated correctness proofs of a sliding window protocol under assumptions of unbounded window sizes, buffer sizes, and channel capacities. We also verify a version of the protocol where the window size is fixed. Since our mechanized target logic is WS1S, not the finite structures of traditional model checking, our method employs only two easy reductions outside the decidable framework. Additionally, we formulate invariants that describe the reachable global states, but the bulk of the detailed reasoning is left to the decision procedure. Because the notation of WS1S is too low-level to describe complicated protocols at a reasonable level of abstraction, we use a higher level language for the protocol description, and then build a tool that automatically translates this language to the MONA syntax. The higher level language we use is IOA.
\end{abstract}

Keywords: Automated Verification, Formal Methods, Sliding Window Protocol, MONA, I/O Automata

\section{INTRODUCTION}

Network protocols are complicated. Protocol developers often determine the validity of their protocols by running simulations. While simu-

"Most of this work was done while the author was at AT\&T Labs Research, NJ, U.S.A..

The original version of this chapter was revised: The copyright line was incorrect. This has been corrected. The Erratum to this chapter is available at DOI: 10.1007/978-0-387-35533-7_26

Tommaso Bolognesi and Diego Latella (Eds.), Formal Methods for Distributed System Development. ๑ 2000 IFIP International Federation for Information Processing.

Published by Kluwer Academic Publishers. All rights reserved. 
lations can be useful, simulations generally cannot show that a protocol is correct for all possible cases. Mechanical formal verification is another way to check the validity of protocols. The traditional benchmark used for testing mechanical verification of protocols is the alternating bit protocol [2]. For this protocol, many papers have shown that mechanical verification is viable (see for example $[4,9,15,1])$.

In this article we demonstrate that an abstract algorithm description language can be effectively linked to the MONA tool $[12,10]$ for verification purposes. In particular, with only a couple of simple abstraction steps, the verification of invariants can be fully automated for network protocols that are substantially more complicated than the alternating bit protocol. We also argue that the state machine description IOA language [6] allows us to express the protocol at a convincing level of abstraction, and we discuss a translator from IOA to MONA.

We use these tools to verify a sliding window protocol. The protocol allows a dynamic and unbounded window size; and it assumes unbounded, lossy channels, and unbounded buffers. Our automated tools also allow us to verify the protocol with a fixed window size, and we verify the protocol with a window size as large as 256 . The proof depends on two simple, meta-logical simplifications in the spirit of abstract interpretation. The proof itself consists of an invariant whose correctness is established automatically by MONA.

The main contribution of our work is to effectively demonstrate that the MONA tool can be used to verify non-trivial protocols with unbounded data structures as well as bounded data structures. Unbounded data structures cannot be handled by traditional Binary Decision Diagrams (BDDs) [3] based tools. On the other hand, theorem provers (for example $[8,16])$ can also handle unbounded data structures. However, given the complexity of the protocol we verify in this work, we believe our verification process requires a lot less user interaction than is typical with theorem provers. By linking the MONA tool with IOA via the translation tool, we also allow users to describe protocols in a reasonably intuitive manner.

\subsection{THE MONA TOOL}

The MONA tool is an efficient implementation of the decision procedures for WS1S (Weak Second-order Logic of One Successor) and WS2S (Weak Second-order Logic of Two Successor). WS1S is a variation of first-order logic with quantifiers and boolean connectives. Its interpretation is tied to a somewhat weakened version of arithmetic. The WS1S first order variables denote natural numbers, which can be compared and 
subjected to addition by constants. The logic also allows second-order variables which can be interpreted as a finite set of numbers. WS2S is a generalization of WS1S interpreted over the infinite binary tree.

A MONA program consists of a number of declarations and formulas. While the WS1S/WS2S logics have a simple and natural notation, the notation is too low-level to define protocols in a readable manner. Thus, we decided to use a high level language for describing the protocols. The protocol written in the high level language would then be translated to an equivalent MONA program.

\subsection{THE IOA LANGUAGE}

We decided to use the IOA language of Garland and Lynch [6] as the high level language. The IOA language is a precise language for describing Input/Output (I/O) Automata and stating their properties. I/O Automata [14], models components in asynchronous concurrent systems as labeled transitions systems. The model provides a mathematical framework for describing and reasoning about system components that interact with each other in an asynchronous manner. An I/O automaton consists of five components, a set of states, a nonempty set of start states, a set of actions, a set of steps, and a optional set of tasks. The set of actions can be partitioned into three disjoint sets, input, output, and internal. The set of tasks is a partition of the output and internal actions of the automaton.

\subsection{RELATED WORK}

The sliding window or go back $n$ protocol is used in various standard data link control (DLC) protocols such as HDLC (high level DCL) and SDLC (synchronous DCL). It also forms the basis of reliable transport layer protocols like TCP. The sliding window protocol has been studied extensively and there are several works that present manual verification of the protocol. For example see $[13,19]$.

There has been a lot less work on mechanical verification of the sliding window protocol. In [18] Richier et al. use temporal logic and model checking to verify safety properties of the sliding window protocol. Because of the state explosion problem, verification is only possible for a fairly restricted set of values of parameters. Another paper in which a mechanical verification of the sliding window protocol is carried out is [11] by Kaivola. In this paper both safety and liveness proofs are performed. In order to alleviate the state explosion problem, Kaivola uses compositional property preserving equivalences and preorders which allows one to replace components of a system with smaller 
ones. The verification can then be carried out on the smaller systems. Using non-divergent failures divergences (NDFD) preorders, safety and liveness properties of the sliding window protocol for arbitrary channel lengths and "realistic" parameter values are verified semi-automatically. The largest parameter value presented in Kaivola's paper is a window size of seven, which is used with two other smaller fixed values that represent buffer sizes. Godefroid and Long [7] verify a full duplex sliding window protocol with the queue size as large as 11. Queue BDDs which they introduce and use in their verification of the protocol are very similar to BDD-represented automata used by the MONA tool.

One way in which our work here differs from the other papers on mechanical verification of the sliding window protocol is that our model of the protocol is different from the others. First, we assume that the sender and receiver have unbounded buffers and that the sender uses an unbounded set of sequence numbers. Because we assume unbounded sequence numbers, we do not require that the channels in our model be FIFO. While the assumption of unbounded buffers and sequence numbers is not a realistic one, in the situation where the sliding window protocol is used in the transport layer of the Internet (a la TCP [17]), the assumption of FIFO channels is not realistic either. TCP does not assume FIFO channels, instead it uses timeout mechanisms to simulate unbounded sequence numbers. This timeout mechanism is quite complicated, so we decide to just assume unbounded sequence numbers. Nevertheless, we believe our model more closely resembles the actual workings of TCP that the models used in the related work discussed above. Another way in which our work differs is that we do not have to assume a fixed window size. Our model allows the window size to be set nondeterministically. However, if we assume a fixed window size, we have verified the protocol with a window size of 256 .

\subsection{ORGANIZATION OF THE PAPER}

In Section 2 we briefly describe the program that translates IOA to the MONA syntax. Section 3 and 4 contain descriptions of two versions of the sliding window protocol with unbounded window sizes, and also describes the abstractions we use. The proof of safety is discussed in Section 5. In Section 6 we discuss the case where we fix the window size, and we also present some experimental results. Section 7 contains some concluding remarks. 


\section{THE TRANSLATOR PROGRAM}

The IOA language is Turing complete. Therefore, we cannot translate every IOA program into a valid equivalent WS1S/WS2S formula. However, we are able to translate a subset of the IOA language that is sufficiently expressive to allow the formulation of reasonable protocol descriptions.

We treat the simple built in types of the IOA language in a straightforward manner. IOA types boolean and (non-negative) integer translate to boolean and integer in WS1S. However, we cannot translate reals, and we do not translate IOA types character or string.

The IOA language also has several built in "compound" types. These types are, Array [I, E], Map [I, E], Seq[E], Set [E] and Mset [E]. For these types, $I$ is the index type, and $E$ is the type of the elements. Each compound type has a set of built in operators. We are able to translate these compound types when the size of $E$ has a known bound. However, for type Set $[E]$, where $E$ is the set of integers, we translate directly to the MONA representation of sets of integers. The other types we represent in MONA as a group of sets where one set contains the valid indices, when the data type has indices, and the other set(s) are used to represent the elements. The IOA language also has an enumeration type. We represent this type in MONA using boolean valued variables, where the bit value of the variables taken together as binary number corresponds to the enumerated values.

We illustrate the translation schema with a small example. If we have an IOA type Animals which is an enumeration of dog, cat, rat, and bat, it is translated to MONA boolean (zero'th-order) variables, say S0 and $S 1$, where the values $S 0=$ false and $S 1=$ false represent dog, $S 0$ = false and $S 1=$ true represent cat, $S 0=$ true and $S 1=$ false represent rat, and $S O=$ true and $S 1=$ true represent bat. A compound IOA variable animalBuf that has type Seq[Animals] is translated into three second order MONA variables, say S2, S3, and S4. The first set S2 holds the set of valid indices for the sequence. The other two sets encode the values in these positions in the following manner: for each valid index in S2 the presence or absence of that index in sets S3 and $\mathrm{S} 4$ encodes the values in the position. Thus, if dog is the value of position 0 in the sequence, then sets S3 and S4 do not contain the value 0 . However, if cat is the value in position 0 , then set S3 does not contain 0 while set $\mathrm{S} 4$ does. Thus, animalBuf $=$ [cat, rat, dog, bat, rat] where cat is at index position 0 is represented by the MONA sets $\mathrm{S} 2=\{0,1,2,3,4\}, \mathrm{S} 3=\{1,3,4\}$, and $\mathrm{S} 4=\{0,3\}$. IOA operators on sequences are translated accordingly. For example, the MONA translation 
of the head operator checks if the value 0 is present in the first set representing the sequence; this is a test for emptiness, and if the sequence is empty head is undefined. If the sequence is not empty, the two boolean values returned by the MONA operations 0 in S3 and 0 in S4 gives the value of the head of the sequence. In this example the values returned would be false and true, which is our MONA representation for cat.

IOA transitions are translated to MONA predicates. We used unprimed and primed MONA variables to represent the pre and post states of the IOA variables. IOA transitions are atomic, but the assignments in a transition happen sequentially. Thus, in our MONA translation we add temporary variables to hold intermediate values. The translator also generates a MONA predicate that represents the assignment of initial values to the IOA variables.

To facilitate the verification process we add a predicate, preds, section to the IOA language constructs. The preds section of an IOA program contains named predicates on the state of the program variables. We translate the predicates to unprimed and primed MONA predicates of the same name. The unprimed state represents the values before a transition and the primed states represent the values after the transition. Two MONA predicates Inv and Inv' that respectively are the conjunction of the unprimed and primed predicates in the preds section are automatically generated. Inv is the Invariant we wish to verify holds for the IOA protocol. The translator also creates a separate file for each transition in the IOA program. Each file contains a MONA predicate

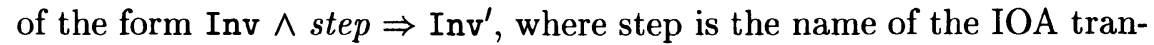
sition. Additionally, a MONA file which contains the MONA formula to test that Inv holds in the initial state is generated. These are the necessary files for the MONA tool to automatically verify the invariant. To verify that the invariant holds, we run MONA on each of the files. If MONA tells us that all the formulas are valid, then the invariant holds.

\section{THE PROTOCOL DESCRIPTION}

In this section we describe our general IOA model of a sliding window protocol. The sliding window protocol is a mechanism for reliable message delivery in a distributed setting where a sender and a receiver communicate over lossy channels. The basic structure of these protocols is shown in Figure 1. In addition to the four components we model, there are two external users. The user on the sender side inputs the data to be sent by the sender, and the user on the receiver side gets the data that the receiver delivers. 


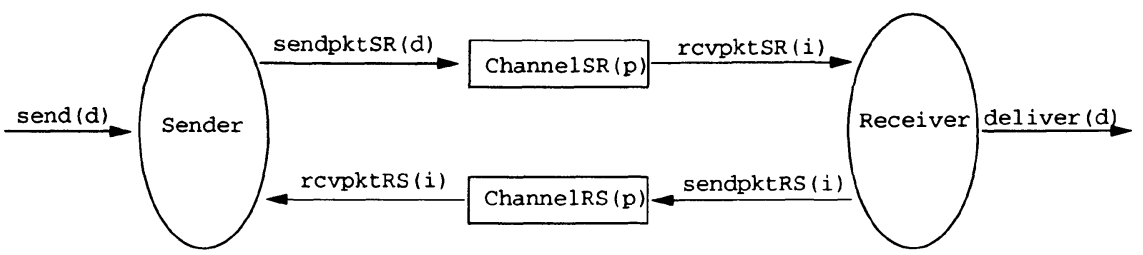

Figure 1 The basic structure and components of a sliding window protocol.

The basic idea of the sliding protocol is that a window of size $n \geq$ 0 determines how many successive packets of data can be sent in the absence of a new acknowledgment. The window size may be fixed or may vary depending on the conditions of the different components of the protocol. In our model we will assume that $n$ varies nondeterministically. Each packet of data is sequentially numbered, so the sender is not allowed to send packet $i+n$ before packet $i$ has been acknowledged. Thus, if $i$ is the sequence number of the packet most recently acknowledged by the receiver, there is a window of data numbered $i+1$ to $i+n$ which the sender can transmit. As successively higher-numbered acknowledgments are received, the window slides forward. The acknowledgment mechanism is cumulative in that if the receiver acknowledges packet $k$, where $k \geq$ $i+1$, it means it has successfully received all packets up to $k$. Packet $k$ is acknowledged by sending a request for packet $k+1$. Typically, transmitted data is kept on a retransmission buffer until it has been acknowledged. Thus, when $k$ is acknowledged, packets with sequence number less than or equal to $k$ are removed from the retransmission buffer. Packets that are not acknowledged are eventually retransmitted. For our modeling of the sliding window protocol we assume that sequence numbers are unbounded, we do not assume that the channels are FIFO.

Below we present two IOA versions of the sliding window protocol. The first version is a general formulation, where the data domain is unspecified. The second version of the protocol, is a parameterized transition system expressible in WS1S; it relies on two additional assumptions: that the data domain consists of two values and that the channels are approximated (in the sense to be explained). This version of the protocol is in the full paper [20].

\subsection{A GENERAL PROTOCOL MODEL}

In this section we define a sliding window protocol as an I/O Automaton. The I/O automaton is defined using the IOA language. The general IOA model is shown in Figure 2. 


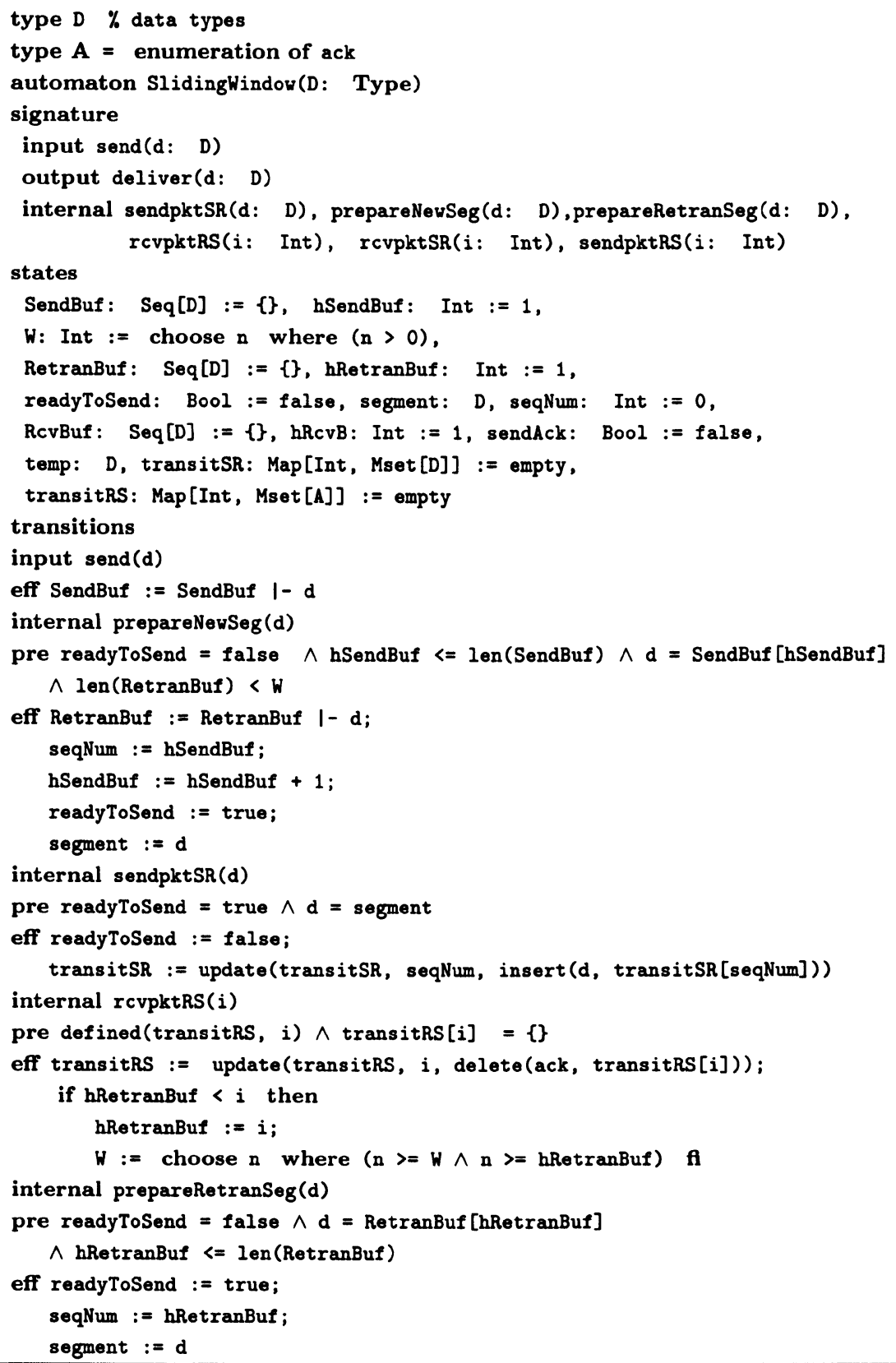

Figure 2 The general IOA model of the sliding window protocol. 
3.1.1 Types. The type D represents a data domain whose internal structure is unspecified. The type $\mathrm{A}$ is an acknowledgment type, and has only one possible value, ack.

3.1.2 Signature and States. The signature is a partition of the actions of the automaton into input, output and internal actions. The actions send(d), sendpktSR(d), prepareNewSeg(d), prepareRetranSeg (d) and rcvpktRS (i) all happen on the sender side. The actions rcvpktSR(i), sendpktRS(i), and deliver(d) happen on the receiver side, and the actions $\operatorname{dropSR}(i)$ and $\operatorname{dropRS}(i)$ happen in the channel from the sender to the receiver and the channel from the receiver to the sender respectively. We explain the functionality of each action when we describe the transitions below.

The states part of the IOA program represents the set of states and the set of initial values. In the listing, variables SendBuf through hRetranBuf are variables of the sender, variables RcvBuf through temp are receiver variables, variable transitSR is the state variable of the channel from the sender to the receiver, and variable transitRS is the state variable of the channel from the receiver to the sender. The variable SendBuf is a sequence that holds elements of type $D$ and is initially empty. This buffer holds the input data, and the position of the element in the sequence corresponds to the sequence number of that piece of data. The buffer has an associated pointer called hSendBuf; the section from hSendBuf to len(SendBuf) is called the unsent part of SendBuf. The variables RetranBuf and hRetranBuf represent the retransmission buffer in the same way the send buffer is represented. The variable $W$ represents the current edge of the window, that is, the highest index allocated for use by the retransmission buffer. The non-deterministic setting of the initial value allows us to have an unbounded window size. Variable readyToSend is a boolean flag. It is set whenever a datum in the segment variable is ready to be transmitted; seqNum is then the sequence number of that segment.

On the receiver side, the variable RcvBuf holds received data before it is passed to the external user. Variable hRcvBuf is the index of the first element of the undelivered part of this buffer. The variable sendAck is a boolean flag that enables the sending of an acknowledgment packet, and temp is a data variable that is used to hold a value received from the channel before it is added to RcvBuf.

The variables transitSR and transitRS are map types that represent packets in transit from the sender to the receiver and from the receiver to the sender respectively. Both maps are indexed by integers. The transitSR map holds elements that are multisets with elements of type 
D. These elements are multisets because there may be duplicates of data packets on the channel. The index of the defined elements in transitSR represents the sequence number of the packet(s). For transitRS the element type is a multiset of type A. The index of an element of transitRS represents the acknowledgment number of the packet.

3.1.3 Transitions. The send (d) input causes the data item $d$ from the external user to be appended to the end of SendBuf. The internal action prepareNewSeg (d) prepares the data item d to be sent. This is data that has not been sent before. The precondition hSendBuf $\leq$ len(SendBuf) means the active part of the send buffer, SendBuf, is not empty. The data item $d$ is the element index by hSendBuf. There must also be space available in the allowable window; that is, hSendBuf $<\mathrm{W}$. The effect of the prepareNewSeg (d) action is to append $d$ to the retransmission buffer, RetranBuf. The sending of this data item in a packet is also enabled by the other assignments. The internal action sendpktSR(d) sends the data item $d$ if readyToSend is true and $d=$ segment. This action assigns readyToSend to false so that no more data is sent until some preparation occurs. An element is placed on the channel by inserting it into the multiset in the position in the transitSR map indexed by seqNum. The internal action rcvpktRS (i) passes an acknowledgment packet from the receiver to the sender. The position of the element in the transitRS map represents the acknowledgment number. Valid acknowledgments (hRetranBuf $<i$ ) cause hRetranBuf to be assigned the value $i$, which effectively removes the acknowledged packets from the active part of RetranBuf. When packets are acknowledged, we also non-deterministically update the value of $\mathrm{W}$, the edge of the window. This new value of $W$ must of course be no less than the previous value, and it must also be no less than the head of the retransmission buffer. The prepareRetranSeg(d) is similar to prepareNewSeg(d) action except that the data item comes from RetranBuf and not SendBuf.

The rcvpktSR(i), deliver (d), sendpktRS (i) actions happen on the receiver side. These are the actions where the receiver receives a packet from the channel, delivers data to the user, and send an acknowledgment respectively. Actions dropSR(i) and dropRS(i) are the actions by the respective channels for dropping a copy of a packet. These actions are not shown here but are in the full paper [20], where they are also described further.

\section{A WS1S SLIDING WINDOW MODEL}

We desire to prove that what comes out is what goes in; that is, for all reachable states: 
Predicate omega For all $i$ if $i \leq \operatorname{len}($ RcvBuf) then SendBuf [i] $=$ rcvBuf [i].

We establish this safety property by exhibiting an invariant that implies omega. However, we are not able to carry out any automated proof of invariance for the general model. Instead, we will bend the general model into an abstract model whose transitions can be represented in WS1S.

\subsection{FIRST SIMPLIFYING ASSUMPTION}

The general model is parameterized with the D type. Our first simplifying assumption is based on idea of data-independence:

Proposition 1 [21] The omega property holds for all reachable states of all abstract programs if and only if it holds for $\mathrm{D}=\{$ white, red $\}$.

Proof: The program itself contains no comparisons of data. Thus, if there is some $D$ and some reachable state that violates the omega property by virtue of SendBuf $[i]=d^{\prime}$ and rcvBuf $[i]=d^{\prime \prime}$ with $d^{\prime} \neq d^{\prime \prime}$, then the same program on the same domain $D$ but with all send $(d)$ actions replaced by send $(\hat{d})$, where $\hat{d}=d$ if $d \in\left\{d^{\prime}, d^{\prime \prime}\right\}$ and, arbitrarily, $\hat{d}=$ $d^{\prime}$ if $d \notin\left\{d^{\prime}, d^{\prime \prime}\right\}$, will result in a reachable state violating the omega property. Thus, a two-value data domain suffices to establish omega for all domains. (This proof is due to Wolper [21].)

The concrete model is the general model, where $\mathrm{D}=\{$ white, red $\}$.

\subsection{SECOND SIMPLIFYING ASSUMPTION}

Our second simplifying assumption is that the channel structure of the concrete model can be simplified so that each sequence number is associated not with a multiset, but with an element in a fixed, finite set. The method is that of abstract interpretation [5], where the computation is modeled in an abstract domain, which approximates the concrete domain.

\subsubsection{The Abstract Domain.}

The abstract domain multiD models a multiset over $D$ by four different values: D_empty represents the empty multiset; D $r$ represents a multiset that contains only red occurrences; D_w represents a multiset that contain only white occurrences; and $D_{\_}$rw represents a multiset that have occurrences of both red or white. This correspondence can be expressed mathematically by an abstraction function $\alpha_{\text {multiD }}$ : Mset $[\{$ red, white $\}] \rightarrow$ MultiD that maps a multiset to an abstract value. Similarly, the correspondence can be expressed by a concretization func- 
tion $\gamma_{\text {multid }}:$ MultiD $\rightarrow$ Set[Mset $[\{r e d$, white $\left.\}]\right]$ that maps an abstract value to the set of corresponding multisets. Note that if $a$ is not D_empty, then $\gamma_{\text {multid }}(a)$ is an infinite set of multisets.

Also for any multiset $M, M \in \gamma_{\text {multid }} \circ \alpha_{\text {multid }}(M)$. In this sense, the abstract domain approximates the concrete values of the multisets. The approximation is not exact, of course, since generally there will be many other multisets in $\gamma_{\text {mult id }} \circ \alpha_{\text {multid }}(M)$ than $M$.

The multisets of the transitRS channel are modeled by the domain multiA whose values are A_empty, denoting the empty multiset, and A_pres, denoting a non-empty multiset.

In the second IOA model, we call a global state $a$ an abstract state. It contains two maps modeling the channels, and each may contain an unbounded number of abstract values denoting multisets.

The concretization functions above define for an abstract state $a$ a set $\gamma(a)$ of states $c$ of the concrete IOA model.

All operations involving the multisets are then modified to work on the abstract values. For example, the dropping of a message as specified in $\operatorname{dropSR}(i)$, where $i$ is a sequence number, involves the deletion of an element $v$ from the multiset transitSR(i). In the WS1S version, the deletion is modeled by changing the multiD value non-deterministically. For example, $\mathrm{D} \_\mathrm{rw}$ is changed to either $\mathrm{D} \_$(there is only one occurrence of a white message, which is dropped), D_w (there are only white occurrences after dropping the single red message), or D_rw (there are still occurrences of both red and white messages after a message has been dropped).

This treatment of the abstractions entails that for any computation in the concrete model $c_{0}, c_{1}, \ldots$, we can find a corresponding computation in the abstract domain $a_{0}, a_{1}, \ldots$, whose states include those of the concrete computation in the sense that $c_{i} \in \gamma\left(a_{i}\right)$. In particular, if $c_{0}$ is the initial state of the concrete model, then we can let $a_{0}$ be the initial state of the abstract model.

Proposition 2 Let $\phi$ be a formula that does not refer to channels. If $\phi$ holds for all reachable states of the abstract model, then $\phi$ holds for all reachable states of the concrete model.

Proof: (1) We omit the details which establish on a transition by transition basis that the abstract operations are such that any concrete computation corresponds to an abstract one. (2) By the restriction on $\phi$, all variables $\phi$ mentions have the same value in $c$ as in $\psi(c)$. Therefore, $\phi$ holds about $c$ if and only if it holds about $\psi(c)$.

The statement of the proposition is implied by (1) and (2). 


\subsection{THE ABSTRACT MODEL}

The abstract model is obtained from a transformation of the concrete model. The IOA version of the abstract model is in the full paper [20]. This model includes a preds section that contains predicates on the state of the automaton. We prove in Section 5, where these predicates are discussed further, that their conjunction is an invariant of the IOA SlidingWindow.

\section{PROOF OF SAFETY}

We use the standard inductive meta-argument for proving safety properties through invariants. We find a formula $\phi_{I}$ that implies the safety property, and we show that $\phi_{I}$ holds for the initial state, and that for every step $\left(s, a, s^{\prime}\right)$ if $\phi_{I}$ holds in state $s$ then it also holds in state $s^{\prime}$. All such proofs are completely automated.

Our main task at hand is to find $\phi_{I}$.

\subsection{THE PREDICATES}

Predicate omega asserts the safety property, but by itself this predicate is not invariant, so a stronger condition is needed. The other predicates are found by a combination of intuition and interaction with the MONA tool. When the MONA tool is run on a predicate that is not an invariant, the counter example provided by MONA gives some indication of what is needed to strengthen the predicate. Typically, the counter example involves some state that intuition tells one is not reachable from any start state. A predicate is then written to verify this intuition. The verification of this new predicate may in turn lead to the formulation of other predicates. The predicates are shown in Figure 3.

We claim the conjunction of all the predicates above is invariant for SlidingWindow. The claim is proved by using MONA to automatically verify the following:

\section{omega $\wedge$ alpha $\wedge$ beta $\wedge$ gamma $\wedge$ delta $\wedge$ epsilon $\wedge$ zeta}

This invariant clearly implies the safety property. The verification of the protocol with unbounded window sizes took 68.26 seconds. The largest number of states generated by the MONA tool during the process was 18886 , and the largest number of BDD nodes was 277859 . We ran the tools on a SUNW,Ultra-5_10. 


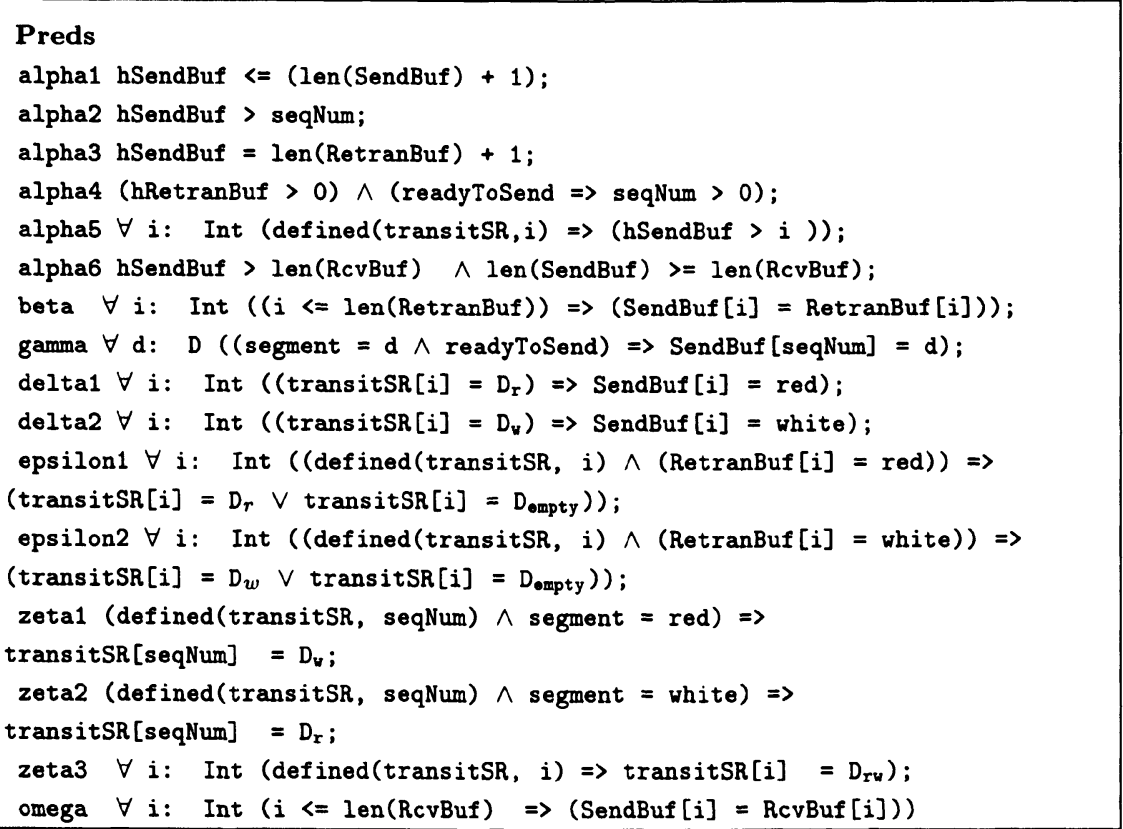

Figure 3 A series of predicates the conjunction of which is invariant on the reachable states of the protocol.

\section{FIXED WINDOW SIZE}

In this section we discuss our results for the protocol where the window size is fixed. We make two changes in the protocol to use a fixed window size. For the prepareNewSed(d) transition the test to check if there is available space in the allowable window is changed to hSendBuf $\leq$ (hRetranBuf +256$)$, where 256 is the fixed window size. For the rcvpktRS (i) transition, the change is removing the update of the variable W.

Table 1 lists our experimental results. In the table, the states and $\mathrm{BDD}$ nodes columns holds the largest number of states and the largest number of BDD nodes generated by the MONA tool during the verification respectively. The results show that the processing time, the largest number of states, and the maximum number of BDD nodes generated by the MONA tool grows linearly as a function of the window size. The linear growth is to be expected: the amount of information that the automaton for the unbounded case carries is bounded (by the size of the state space). The automaton for the bounded case must, in addition, count up to the window size, and that makes the resulting automaton 
size linear in the window size. In our experiments we note that the size of the automaton and the running time in the bounded case surpasses that of the unbounded case for window sizes greater than 44 .

Table 1 The results of our experiments verifying the protocol with different fixed window sizes.

\begin{tabular}{llll}
\hline window size & time (sec's) & states & BDD nodes \\
\hline 16 & 30.98 & 4838 & 147223 \\
32 & 46.67 & 5983 & 258583 \\
64 & 86.63 & 11103 & 481303 \\
128 & 342.01 & 21343 & 926743 \\
256 & 1286.45 & 41823 & 1817623 \\
\hline
\end{tabular}

\section{CONCLUSION AND FUTURE WORK}

In this paper we have demonstrated that a decision procedure for WS1S can be used to verify safety properties of a non-trivial network protocol.

Although our work indicates that for network protocols a good deal of nitty-gritty reasoning about queues and indices can be carried out automatically, more research into the following problems is needed: handling of sequence numbers that wrap around modulo the length of the window; handling of a more concrete presentation of queues, such as those actually found in a real programming language; automated support for abstraction steps; and handling of liveness properties.

\section{Acknowledgments}

Thanks to Dennis Dams and Richard Trefler for helpful comments.

\section{References}

[1] P.A. Abdulla, A. Aniinichini, S. Bensalem, A. Bouajjani, P.Habermehl, and Y. Lakhnech. Verification of infinite-state systems by combining abstraction and reachability analysis. In Computer Aided Verification. 11th International Conference, CAV'99, volume 1633 of LNCS. Springer-Verlag, July 1999.

[2] K. A. Barlett, R. A. Scantlebury, and P. C. Wilkinson. A note on reliable transmission over half duplex links. Communications of the ACM, 12, 1969.

[3] R. E. Bryant. Symbolic boolean manipulation with ordered binary-decision diagrams. ACM Computing Surveys, 24(3):293-318, September 1992.

[4] E.M. Clarke and E.A. Emerson. Design and synthesis of synchronization skeletons using branching time temporal logic. In Workshop on Logics of Programs, 
volume 131 of LNCS. Springer-Verlag, 1981.

[5] P. Cousot and R. Cousot. Abstract interpretation: A unified lattice model for static analysis of programs by construction or approximation of fixpoints. In Proceedings of 4th ACM Symposium on Principles of Programming Languages, pages 238-252, Los Angeles, California, 1977.

[6] Stephen J. Garland and Nancy A. Lynch. The IOA language and toolset: Support for designing, analyzing, and builiding distributed systems. Technical Report MIT/LCS/TR-762, M.I.T., August 1998.

[7] P. Godefroid and D.E. Long. Symbolic protocol verification with Queue BDDs. Formal Methods in System Design, 14(13):257-271, may 1999.

[8] M. J. C. Gordon and T. F. Melham, editors. Introduction to HOL: A Theorem Proving Environment for Higher Order Logic. Cambridge University Press, 1993.

[9] K. Havelund and N. Shankar. Experiments in theorem proving and model checking for protocol verification. In Formal Methods Europe (FME), volume 1051 of Lecture Notes in Computer Science. Springer-Verlag, July 1996.

[10] J. G. Henriksen, J. Jensen, M. Jørgensen, N. Klarlund, R. Paige, T. Rauhe, and A. Sandholm. Mona: Monadic second-order login in practice. In Tools and Algorithms for the Construction and Analysis of Systems, First International Workshop, TACAS '95 LCNS 1019, 1995.

[11] Roope Kaivola. Using compositional preorders in the verification of sliding window protocol. In Computer Aided Verification. 9th International Conference, CAV'97, volume 1254 of $L N C S$, pages 48-59, Haifa, Israel, June 1997. SpringerVerlag.

[12] Nils Klarlund. Mona \& Fido: The logic-automaton connection in practice. In CSL '97 Proceedings, volume 1414 of LNCS. Springer-Verlag, 1998.

[13] Donald Knuth. Verification of link-level protocols. BIT, 21:31-36, 1981.

[14] Nancy Lynch and Mark Tuttle. An introduction to input/output automata. CWI Quarterly, 3(2), September 1989.

[15] Panagiotis Manolios, Kedar Namjoshi, and Robert Sumners. Linking theorem proving and model-checking with well-founded bisimulations. In Computer Aided Verification. 11th International Conference, CAV '99, volume 1633 of LNCS. Springer-Verlag, July 1999.

[16] S. Owre, J. Rushby, N. Shankar, and F. von Henke. Formal verification for faulttolerant architectures: Prolegomena to the design of PVS. IEEE Transactions on Software Engineering, 21(2):107-125, 1995.

[17] Jon Postel. Transmission Control Protocol - DARPA Internet Program Specification (Internet Standard STC-007). Internet RFC-793, September 1981.

[18] J. L. Richier, C. Rodriguez, J. Sifakis, and J. Voiron. Verification in Xesar of the sliding window protocol. In Protocol Specification, Testing and Verification VII, pages 235-248. North-Holland, 1987.

[19] A. Udaya Shankar and S. S. Lam. An HDLC protocol specification and verification using image protocols. ACM Transactions on Computer Systems, 1(4):331$368,1983$.

[20] Mark A. Smith and Nils Klarlund. Verification of a sliding window protocol using IOA and MONA. Technical report, IRISA. To appear.

[21] P. Wolper. Synthesis of communication processes from temporal logic specifications. In Proceedings 13th ACM Symposium on POPL, pages 184-193, January 1986 . 